\title{
The Incedance Of Hydatid Cyst In Human In Baghdad Governorate
}

\author{
*Dr. Maysara.S .Khalf, ** Dr.Lazim H.AlTaie, *** Dr. Mohammad A. \\ AlFaham. \\ *Assi.lecturer College of Medical \& Health Technology. \\ ** Assit .Prof.College of Medicine /Al-Nahrain university. \\ *** Assit. Prof.College of Medicine / Baghdad university.
}

\begin{abstract}
:
Background: Cystic echinococcosis or hydatidosis is caused by the larval stage of the tapeworm Echinococcus granulosus, is one of the most important parasitic infestations in livestock worldwide and one of the most important parasitic zoonoses. A crosssectional study was conducted to estimate the prevalence of hydatidosis in humans in Baghdad governorate and determine the risk factors associated with disease occurrence.

Methods: After collecting hydatid cysts from patients was done to determine the presence of hydatid cysts in human. The viability and fertility tests were done on all of the cysts collected during the study. A questionnaire survey to identify risk factors of transmission was also carried out.

Results: A total of sixty (60) cases of human hydatidosis were collected,(73\%) were liver cyst,(20\%) were lung cyst, (3\%) cyst were in liver and lung and (3\%) were liver, lung and spleen .Our result was showed after viability examination,,$(47 \%)$ of cyst were fertile,(37\%)cyst were steriale,(10\%) cyst were calcified and (7\%) cyst were under developed.sixty (60\%) were femals and (40\%) were males.

Conclusion: The study has established that hydatidosis is prevalent in humans in Baghdad governorate and that the risk factors for its transmission exist. The disease health concern

Keywords: hydatid cyst, prevalence rate, vibility test, Baghdad governorate .
\end{abstract}

\section{Introduction}

Hydatidosis/cystic echinococcosis (CE) is a severe zoonosis caused by the larval stages of a cyclophyllidean cestode called Echinococcus granulosus. Two hosts are involved in the completion of the life cycle of E. granulosus. The definitive hosts are carnivores which harbour mature tape worms in the intestine $(1,2)$. The intermediate hosts of E. granulosus include ungulates both domestic and wild animals and humans. The adult worm lives in the small intestine of carnivores (definitive host), and the intermediate larval stage (Hydatid cyst or Hydatid) develops in the internal organs of a wide range of mammalian species such as goats, sheep and cattle, including humans, which acquire the infection through accidental ingestion of the tapeworm

eggs (3)

Cystic Echinococcosis is known to be one of the most important parasitic infections in livestock worldwide and one of the most widespread parasitic zoonoses $(4,5)$.

The disease has a worldwide distribution and is endemic in many countries of the Mediterranean basin, North and East Africa, Western and Central Asia, China, South America and Australia $(6,7)$

However, even if the distribution of Echinococcus granulosus is considered

worldwide, it is higher in developing countries, especially in rural communities where there is close contact between dogs and various domestic animals (3).

In some western countries, $\mathrm{CE}$ is being considered a remergingzoonosis, due to recent increases in the observed prevalence $(8,9)$.

In humans, $\mathrm{CE}$ is the most common presentation and probably accounts for more than

$95 \%$ of the estimated 2 to 3 million global cases $(10,11)$

The echinococcosis/hydatidosis has considerable socioeconomic impact in both human and animal health in different countries (12). In humans, after a long latency period, the disease consequences may include; poor quality of life (disability adjusted life years [DALYS]); costs of medical treatment, lost opportunity for income generation and mortality in some cases(11) while in animals there is reduced productivity and monetary losses due to abattoir condemnations $(13,14)$. The DALYS for human CE was recently estimated to be more than that for onchocerciasis and almost the same as that for Africa trypanosomaisis(15).

Transmission and maintenance of echinococcosis is dependent on complex

interactions of several factors, including environmental, host and pathogen factors. A number of such factors are of local epidemiological significance and the identification of such factors is important in the effective implementation of control strategies In Iraq, echinococcosis has been reported to occur in many 
parts of the country.(16,17). The aim of this study was to determine the prevalence of the disease in humans.

The study also sought to identify risk factors predisposing infections (11).

\section{Samples collection:- Hydatid fluid collection:-}

\section{Material and Method}

Twenty to fifty $\mathrm{ml}$ of hydatid fluid was aspirated directly from the cyst during operation before injection of any scolicidal agent in to the cyst. Samples were collected from Al-Khadmyia Hospital from the period (1/2/2013$1 / 8 / 2013$ ) and then transferred into caped plastic tubes. The hydatid fluid from the liver and lungs was aspirated under aseptic conditions

Examination of Hydatid cyst fertility and viability: A total of (60) hydatid cysts were examined, hydatid fluid samples were aspirated directly from the cysts during operation before injection of any scolicidal agent into the cyst from the different organs of each patient,particularly lung, liver,and spleen,. Each hydatid cyst was examined visually and manually (palpation and incision). Each individual cyst was aspirated directly from the cyst and was carefully incised and examined for protoscoleces, which look like white dots on the germinal epithelium; such cysts were characterized as fertile cysts. Fertile cysts were subjected Crude hydatid cyst antigens for diagnosis of hydatosis in human(18). To a fertility test. Protoscolices were removed by centrifugation(centrifuge:LKB,Macrospin R,Sweden) at $4^{\circ} \mathrm{C}, 400 \mathrm{Xg}$ for 10 minute.The sediment was then collected and film was made,between slide and cover slip and then examined under the low power magnification (10X) and high power magnification (40X) of the light microscope (Zeiss,Germany). On the other hand the fluid from several fertile cysts was pooled. The supernatant was collected as crude hydatid cyst fluid antigen in vials and stored at $-20^{\circ} \mathrm{C}$. To a viability test. A drop of the sediment containing the protoscoleces was placed on a microscope glass slide,covered with a cover slip and observed with a (40X) objective lens for amoeboid-like peristaltic movements(18

\section{Results}

Sixty (60) cases of human hydatidosis, decidedly surgically confirmed in Bagdad ,having contracted hydatodosis.Age of the patient varied between less than 10 years to above 60 years the highest surgical prevalence of the disease was found in the agegroup (10-19) years.Table (1).

Table (2)Age Distribution In Patients With Hydatid cyst .

\begin{tabular}{rrrr}
\hline Age Group /years & Number of cases & Percentage (\%) \\
\hline Less than 10 & 4 & 14 & 23.3 \\
$\mathbf{1 0 - 1 9}$ & $\mathbf{2 0 - 2 9}$ & 10 & 16.6 \\
$\mathbf{3 0 - 3 9}$ & 12 & 20 \\
$\mathbf{4 0 - 4 9}$ & 8 & 13.3 \\
$\mathbf{5 0 - 5 9}$ & 10 & 2 & 16.6 \\
$\mathbf{6 0}$ and above & 3.3 \\
Total & 60 & 100 \\
\hline
\end{tabular}

The Sixty (60\%) of patients were femals and fourty (40\%) cases were males table (2).Hydatidosis apper more effective in femals than males .

Table(2): Distribution of patients According to Their Gender .

\begin{tabular}{|c|c|c|c|}
\hline Gender & Number & Percentage & $(\%)$ \\
\hline Females & 36 & & $60 \%$ \\
\hline Males & 24 & & $40 \%$ \\
\hline Total & 60 & & $100 \%$ \\
\hline
\end{tabular}

In our result the sixty cases of hydatid cyst were in different organs (44)cyst were found in the liver ,12 cyst may found in the lung,2cyst may found in the liver and lung and 2 cysts were in the liver, lung and spleen .table (3).

Table(3): The Distribution of hydatid cyst in Infected patients .

\begin{tabular}{rrrr}
\hline Hydatid cyst location & Number & Percentage (\%) \\
\hline Liver & 44 & \\
Lung & 12 & 20 \\
Liver+lung & 2 & 3.3 \\
Liver+lung+spleen & 2 & 60 \\
\hline
\end{tabular}


Further more,all the hydatid cyst collected were examined by the viability test to detected steriale,(10\%)were found calicefied and finally (7\%)of hydatid cyst were under developed. Table(4): The Incidence of Hydatid cyst ,Types in Human Isolate.

\begin{tabular}{|l|r|r|r|r|r|}
\hline Species & No.of cyst & No.of fertile & No.of Sterile & No.of Calcified & \multicolumn{2}{|c|}{ No.of under developd } \\
\hline $\begin{array}{l}\text { Human } \\
\text { isolates }\end{array}$ & 60 & $28(46.6 \%)$ & 22 & 6 & 4 \\
$(10 \%)$ & $(36.6 \%)$ & $(6.6 \%)$ \\
\hline
\end{tabular}

\section{Discussion :}

Hydatidosis, caused by Echinococcus spp. (E. granulosus and E. multilocularis) in Iraq is one of the most important zoonotic diseases, throughout the most parts of the world. Ingesting embryonated eggs through hands, food, drinks or material contaminated with parasite eggs infects humans; the larvae reach the blood and lymphatic circulation and transport to the liver, lungs and other organs (19). The asymptomatic period is too long and the disease might be diagnosed even after 20-25 years post infection Cystic echinococcosis is considered endemic in the entire Mediterranean zone including all countries from the Middle East, but alveolar echinococcosis is less prevalent and has been reported only from Iran, Turkey, Iraq and Tunisia(20).

According to Table 1, and in coincidence with global evidence, hydatidosis in Iraq, has no privilege for age. Hydatidosis is a disease of long incubation period (might be 20 to $30 \mathrm{yr}$ ) and accordingly a wide range of different ages is obvious in infected patients. (20).

The overall our result shows the range of 10-59 years old as the age group of the highest cases, Women have the highest chance of contact with sources of infection such as dog, soil, vegetable etc, yet in some areas depending on the culture and social criteria it is possible that men cover the highest rate of infection.(19) Housewives, especially in rural areas, where the most infected cases can be found, have the highest chance of contact with the sources of infection. Contact with contaminated vegetables, cleaning the house containing the dog faeces, desire to eat soil (Geophagy) as longing in pregnant embrace the etiological issues,Highly infection rate in females is in agreement with most of other studies which have shown a high frequency in females $(21,22)$. The highest risk group in the country is specifically in Arab Gulf region -in general- which are women and children. Traditionally, rural women still bear the biggest burden of tending animals - whether breeding, milking, or wool -shearing- and domestic or stray dogs are never faraway. The added chore of women preparing and cooking contaminated food and vegetables with little clean water at hand increases considerably the risk of infection. In many parts of Middle East during springtime, it is common practice together berries and various wild plants which are eaten unwashed and geophagia among children and pregnant women is well known. infection rates among women are shown to be the highest and children who acquire the disease in early life may not present with symptoms until adulthood (23). Also, estrogen hormone plays an important role in dissolve egg shells and facilitating hatched Oncosphere to penetrate host tissues in females of mice (24). However, this result does not agree with the findings of Torgerson et al. (2003) (25)who observed high rates of infection in males.

Hydatid disease is characterized by cystic space - occupying lesions in the liver, lungs and rarely in other parts of the body (26). All evidences provided ensure that hydatid disease till now major health problem in Iraq in spite of modern equipments available for diagnosis and treatment. The surgically confirmed cases are the only reliable source of data on human hydatidosis, since hydatid infection is a notifiable disease, and it is difficult to determine the specific source of infection and its usually impossible to know when the infection was acquired this may be due to the fact that cysts are usually slowly growing and the development of symptoms or the ability to diagnose the conditions may require from 6 months to several years after exposure to the infections (27).

The liver act as the first filter for larval infection and the lung acts as the second filter. Distribution of infection in different organs showed that the liver was the most frequently involved $44(73.3 \%)$ when compared with lung $12(20 \%)$ and liver and lung 2(3.3\%) and other multiple infected organs such asliver ,lung and spleen $2(3.3 \%)$ Table 3, generally these proportions approximately in agreement with most of previously recorded data by Ahmadi and Al-Dalimi (2006) (28) .

Fertile Hydatid cysts are formed in intermediate hosts (human and herbivores) producing protoscolices, the infective form to canines, at their germinal layers. Infertile cysts are also formed, but they are unable to produce protoscolices. The molecular mechanisms involved in hydatid cysts fertility/infertility are unknown. Nevertheless, previous work has suggested that apoptosis is involved in hydatid cyst infertility and death. On the other hand, fertile hydatid cysts can resist oxidative damage due to reactive oxygen and nitrogen species. On these foundations, they have hypothesized that when oxidative damage of DNA in the germinal layers exceeds the capability of DNA repair mechanisms, apoptosis is triggered and hydatid cysts infertility occurs. Fertility of hydatid cyst is important factor in stimulation of immune response in patients with hydatidosis as observed in case of increasing of IgG and IgM concentrations when there's daughter vesicles within cyst and this factor directly proportional with advanced ages showed in Table 4 as we found there 28(46.6\%)were fertile,22(36.6\%)were sterile,6(10\%) calcified cyst and 4(6.6\%) hydatid cyst were under developed .(28). 
Further more, our result shows the risk factors associated with hydatid cyst infection, Vegetable: Here is tendency to eat raw vegetables with food. Unfortunately, in most vegetable farms, dogs freely prowl and contaminate the vegetables. Another potential source of infection is carrot juice, which almost everywhere is sold in a mixture with ice cream. The washing system of carrots is such that the parasites' eggs are retained in washing water and most of them are not separated from the carrots (28). Geophagy: A tendency to eat soil by pregnant women especially in rural areas makes the situation in benefit of infecting with hydatidosis. Children also play a game entails them to contact with soil. (28).

Sheep: Vicinity of sheep with dogs in places selling sheep results to contamination of sheep wool with parasite's eggs. Sheep sellers contact them and while counting money, eating or drinking, ingest the eggs. Shepherds also can be infected in this manner. Other risk factors Contacting with dog and breast pumping are two other great risk factors here.(28).

\section{References}

[1]. Zhang, W., Li, J. \& McManus, D. P. Concepts in immunology and diagnosis of hydatid disease.Clin Microbiol Rev, . (2003) 16, 18-36.

[2]. Khuroo, M. S. Hydatid disease: current status and recent advances. Annals of Saudi Medicine, (2002). $22,56-64$.

[3]. Eckert, J. \& Deplazes, P. Biological, epidemiological, and clinical aspects of echinococcosis, a zoonosis of increasing concern. Clinical Microbiology Review, (2004) 17, 107-35.

[4]. Craig, P. S., Rogan, M. T. \& Allan, J. C. Detection, screening and community epidemiology of taeniid cestode zoonoses: cystic echinococcosis, alveolar echinococcosis and neurocysticercosis. Advances in Parasitology, (1996). 38, 169-250.

[5]. Cabrera, P. A., Lloyd, S., Haran, G., Pineyro, L., Parietti, S., Gemmell, M. A., Correa, O., Morana, A. \& Valledor, S. Control of Echinococcus granulosus in Uruguay: evaluation of different treatment intervals for dogs. Veterinary Parasitology, . (2002), 103, 333-40.

[6]. Jenkins, D. J. Hydatid control in Australia: where it began, what we have achieved and where to from here. International Journal of Parasitology, (2005). 35, 733-40.

[7]. Romig, T., Thomas, D. \& Weible, A. K. Echinococcus multilocularis - a zoonosis of anthropogenic environments? Journal of Helminthology, (2006) 80, 207-12.

[8]. Himsworth, C. G., Jenkins, E., Hill, J. E., Nsungu, M., Ndao, M., Andrew Thompson, R. C., Covacin, C., Ash, A., Wagner, B. A., Mcconnell, A., Leighton, F. A. \& Skinner, S. Emergence of sylvatic Echinococcus granulosus as a parasitic zoonosis of public health concern in an indigenous community in Canada. American Journal Tropical Medicine Hygiene, (2010) 82, 643-5.

[9]. Budke, C. M., Campos-Ponce, M., Qian, W. \& Torgerson, P. R. A canine purgation study and risk factor analysis for echinococcosis in a high endemic region of the Tibetan plateau. Veterinary Parasitolology, (2005). 127, 43-49.

[10]. Craig, P. S., Rogan, M. T. \& Allan, J. C. Detection, screening and community epidemiology of taeniid cestode zoonoses: cystic echinococcosis, alveolar echinococcosis and neurocysticercosis. Advances in Parasitology, (1996). 38, 169-250.

[11]. Budke, C. M., Deplazes, P. \& Torgerson, P. R. Global socioeconomic impact of cystic echinococcosis. Emerging Infectious Diseases, (2006). 12, 296-303.

[12]. Rkia Azlaf, A. D. Epidemiological study of the cystic echinococcosis in Morocco. Veterinary Parasitology, (2006) 137, 83-93.

[13]. Torgerson, P. R. \& Heath, D. D. Transmission dynamics and control options for Echinococcus granulosus. Parasitology, (2003) 127 Suppl, S143-58.

[14]. Scala, A., Garippa, G., Varcasia, A., Tranquillo, V. M. \& Genchi, C. Cystic echinococcosis in slaughtered sheep in Sardinia (Italy). Veterinary Parasitology, (2006) 135, 33-8.

[15]. Budke, C. M., Deplazes, P. \& Torgerson, P. R. Global socioeconomic impact of cystic echinococcosis. Emerging Infectious Diseases, (2006). 12, 296-303.

[16]. Ihsan E. Al-Saimary, Maani N. Al-Shemar, and Mohammed M. A. Al-FayadhEpidemiological and immunological findings on human Hydatidosis. ,(2010) Vol. 1(2), pp. 26-34, November 2010. Medical Practice and Review

[17]. Mahdi M. Bajalan:" Prevalence of echinococcus in stary dog and slaughtered livestock Kalar district/Sulaimaniya province /Kurdistan Iraq",MSc.thesis ,College of veterinary medicine ,University of Baghdad (2006).

[18]. Lemu Golassa, Tamrat Abebe and Asrat Hailu.(2010). Evaluation of crude hydatid cyst fluidantigens for the serological diagnosis of hydatidosis in cattle. Department o Medical Laboratory Sciences and Pathology, College of Public Health and Medical Sciences, Jimma University, PO Box 378, Jimma,Ethiopia: Microbiology, Immunology and Parasitology Department,Medical Faculty, Addis Ababa University, PO Box 9086, Addis Ababa,Ethiopia.

[19]. Muller R. Worms and human diseases. 2nd ed. Wallingord: CABI International, Oxon, UK; 2002.

[20]. Sadjjadi SM. Present situation of echinococcosis in the Middle East and Arabic North Africa. Parasitol Int. 2006;55 Suppl:S197202

[21]. Abdul-Karim H. Hydatid disease of the liver and its biliary communication. Iraq. J. Med. Sci., (2001). 1: $200-203$.

[22]. Al-Qadhi BN. Study of some immunological and biochemical aspects of patients infected with hydatidosis . Ph.D. Thesis. College of Science, University of Baghdad (2005).

[23]. Nakao M, McManus DP, Schantz PM, Craig PS, Ito AA. Molecular phylogeny of the genus Echinococcus inferred from complete mitochondrial genomes. Parasitology, (2007).134: 713-722.

[24]. Brunetti E, Gulizia R, Garlaschelli AL, Filice C. Cystic Echinococcosis of the liver associated with repeated international travels to endemic areas. J. Travel. Med., (2005).12(4): 225-228.

[25]. Torgerson, P. R. \& Heath, D. D. Transmission dynamics and control options for Echinococcus granulosus. Parasitology, (2003). 127 Suppl, S143-58.

[26]. Fakhar M, Sadjjadi SM. Prevalence of hydatidosis in slaughtered herbivores in Qom Province, central part of Iran. Veterinary Research Communications. (2007);31(8):993- 997.

[27]. Daryani A, Alaei R, Arab R, Sharif M, Dehghan MH, Ziaei H. The prevalence, intensity and viabilitymof hydatid cysts in slaughtered animals in the Ardabil Province of Northwest Iran. J Helminthol; (2007) . 81:13-17.

[28]. Ahmadi NA. Hydatidosis in camels (Camelus dromedarius) and their potential role in the epidemiologyof Echinococcus granulosus in Iran. J Helminthol.; (2005 ) ,79(2):119-25.

[29]. Rokni MB. The present status of human helminthic diseases in Iran. Ann Trop Med Parasitol. (2008) ,102(4):283-295. 